\title{
Crystal Structures of Dense Lithium: A Metal-Semiconductor-Metal Transition
}

\author{
M. Marqués, ${ }^{1}$ M. I. McMahon, ${ }^{1}$ E. Gregoryanz, ${ }^{1}$ M. Hanfland, ${ }^{2}$ C. L. Guillaume, ${ }^{1}$ C. J. Pickard, ${ }^{3}$ \\ G. J. Ackland, ${ }^{1}$ and R. J. Nelmes ${ }^{1}$ \\ ${ }^{1}$ SUPA, School of Physics and Astronomy, and Centre for Science at Extreme Conditions, The University of Edinburgh, \\ Mayfield Road, Edinburgh, EH9 3JZ, United Kingdom \\ ${ }^{2}$ European Synchroton Radiation Facility, BP 220, 38043 Grenoble, France \\ ${ }^{3}$ Department of Physics and Astronomy, University College London, Gower Street, London WC1E 6BT, United Kingdom
} (Received 27 August 2010; published 1 March 2011)

\begin{abstract}
$A b$ initio random structure searching and single-crystal $x$-ray diffraction have been used to determine the full structures of three phases of lithium, recently discovered at low temperature above $60 \mathrm{GPa}$. A structure with $C 2 \mathrm{mb}$ symmetry, calculated to be a poor metal, is proposed for the $o C 88$ phase $(60-65 \mathrm{GPa})$. The $o C 40$ phase $(65-95 \mathrm{GPa})$ is found to have a lowest-enthalpy structure with $C 2 c b$ symmetry, in excellent agreement with the $x$-ray data. It is calculated to be a semiconductor with a band gap of $\sim 1 \mathrm{eV}$ at $90 \mathrm{GPa}$. $o C 24$, stable above $95 \mathrm{GPa}$, has the space group Cmca, and refined atomic coordinates are in excellent agreement with previous calculations.
\end{abstract}

DOI: 10.1103/PhysRevLett.106.095502

PACS numbers: 61.50.Ks, 61.05.cp, 62.50.-p, 71.20.-b

Lithium is the simplest metal in the periodic table, and its electronic structure is well explained by the nearly freeelectron model. However, at high pressures in simple metals, the free-electron picture breaks down, through reduced valence volume, pseudogap opening at the Fermi surface, or electride formation [1-4]. This implies lowered crystal symmetry and electrical conductivity, and diffraction studies [5] have revealed a sequence of phases with increasing complexity, up to $c I 16$ a soft-mode distortion of bcc with a 16 atom unit cell [2]. Density functional theory (DFT) showed that a 24-atom orthorhombic structure ( $\mathrm{Cmca}$-24) has lower enthalpy than the $c I 16$ phase above $88 \mathrm{GPa}[6]$ or $100 \mathrm{GPa}$ [7]. A subsequent study using diffraction and electrical resistance measurements at $25 \mathrm{~K}$, along with visual observations, revealed two further structural transitions at $\sim 70$ and $\sim 80 \mathrm{GPa}$ at $25 \mathrm{~K}$, first to a metallic Li-VI phase and then to a semiconducting Li-VII phase [8].

Subsequent DFT studies $[7,9,10]$ attempted to predict the structures of Li-VI and Li-VII. Yao et al. [9] proposed 24-atom insulating structures at 74 and 91 GPa. Pickard and Needs [10] predict that the Cmca-24 structure of Rousseau et al. [6] is stable only above $106 \mathrm{GPa}$, suggesting yet another 24-atom orthorhombic structure (space group $P b c a$, a distortion of the Cmca-24 structure) at $\sim 86 \mathrm{GPa}$, followed by a transition to Yao's $C 2 c b-24$ [11] structure at $98 \mathrm{GPa}$.

Our recent x-ray diffraction studies mapped out the lithium phase diagram in a wide pressure and temperature range [12]. At $200 \mathrm{~K}$, the study located the transitions from cI16 to Li-VI and then to Li-VII at 60 and $65 \mathrm{GPa}$, and also found a further transition to a previously unreported Li-VIII phase at $95 \mathrm{GPa}$. Li-VI, VII, and VIII were reported to have $C$-face centered orthorhombic structures with 88, 40, and 24 atoms (denoted $o C 88, o C 40$, and $o C 24)$, respectively, on the assumption of continuity of atomic volume through the sequence of phase transitions. However, due to the difficulty associated with collecting high-quality data at low temperatures, the detailed structures were not determined on a purely experimental basis. Also, the large size of the $o C 88$ and $o C 40$ unit cells means that previous calculations have been unable to identify these structures correctly.

In this Letter, we combine $a b$ initio random structure searching (AIRSS) [13] and analysis of single-crystal diffraction data to investigate the structures and natures of these three phases. For the description of the experimental and theoretical techniques involved in this study we refer the reader to Refs. $[12,14,15]$ and supplemental online materials [16]. A schematic representation of the $P-T$ space and sequence of the solid phases involved in this study is shown in Fig. 1.

We start by considering the 24-atom structure. Experimentally, all the reflections for the $o C 24$ structure at $125 \mathrm{GPa}$ and $300 \mathrm{~K}$ were indexed with lattice parameters $a=7.424(3) \AA, b=4.175(4) \AA$, and $c=4.214(3) \AA$ and showed systematic absences corresponding to Cmca or $C 2 c b$ space group symmetry, as also found at $105 \mathrm{GPa}$ [16]. Least-squares refinement yielded a structure corresponding to the Cmca-24 structure of Rousseau et al. $[6,10]$ with atomic coordinates in excellent agreement with the calculated values. Our calculations detected the same Cmca, C2cb, and Pbca candidates as previous works; these diverge in enthalpy only in a small pressure region, calculated as 90-95 GPa. Details of the space group determination, structure refinement, and calculations are given in supplemental material [16]).

Li-VII (oC40) is the only known phase of lithium showing pronounced semiconducting behavior (see below and Refs. [8,12]). Single crystals of Li-VII (at $75 \mathrm{GPa}$ and $240 \mathrm{~K}$ ) were of poorer quality than those obtained for 


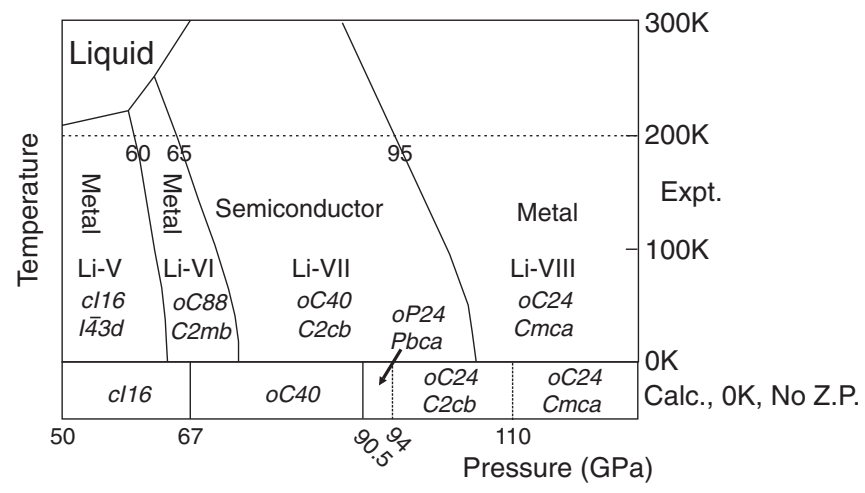

FIG. 1. Diagram showing phases in $\mathrm{Li}$ (adopted from Ref. [12]). Experiments reported here were conducted at $240 \mathrm{~K}(o C 88$ and $o C 40)$ and at $300 \mathrm{~K}(o C 24)$, total energy calculations are effectively at $0 \mathrm{~K}$, and neglect zero-point motion. Numbers show measured and calculated transition pressures in $\mathrm{GPa}$. Low pressure bcc (to $7.5 \mathrm{GPa}$ ), fcc (to $41 \mathrm{GPa}$ ), and rhombohedral $h R 1$ (to $42 \mathrm{GPa}$ ) are not shown.

$o C 24$, resulting in broader, weaker reflections (see [16]). The space group was determined as either $C m c a$ or $C 2 c b$, and least-squares fitting of reflection $d$ spacings gave lattice parameters of $a=4.942(4) \AA, b=8.026(10) \AA$, and $c=6.784(3) \AA$. Searches for possible $o C 40$ structures were initially conducted using a 20 -atom primitive cell with space group $P 2_{1} / c$ (a subgroup of $C m c a$ ). The initial lattice parameters were chosen as the experimental values, but allowed to change during the optimizations. After searching 250 possible structures, the lowest-enthalpy candidate structure with $\mathrm{Cmca}$ symmetry was found to have only a small range of stability against $c I 16$ (see [16]). We then performed 600 further trials with 20-atom structures with three lower-symmetry space groups: $P c$ (subgroup of both $C m c a$ and $C 2 c b$ space groups); randomly selected space groups with 4 and 2 symmetry operations; and symmetry reduced to $P 1$. From these we found a still-lower enthalpy structure with noncentrosymmetric space group $C 2 c b$ and five nonequivalent $\mathrm{Li}$ atoms located on $8 b$ sites ( labeled Li1-5, see [16]).

Calculations of the zone center $(\Gamma)$ phonons for the calculated $\mathrm{Cmca}-40$ structure revealed an imaginary frequency for a $B_{3 u}$ mode, the first-order parameter for the Cmca-40 to $C 2 c b-40$ group-subgroup distortion. Detailed enthalpy calculations (Fig. 2) show that the $C 2 c b-40$ structure also has considerably lower enthalpy than the monoclinic C2-24 structure previously reported to be stable in this pressure range [9]. The predicted $C 2 c b-40$ to Cmca-24 transition pressure is $91.3 \mathrm{GPa}$, in agreement with the experimental value of $95 \mathrm{GPa}$ at $200 \mathrm{~K}$. In fact, our calculations predict that the $C 2 c b-40$ structure would transform to the Pbca-24 structure at $90.5 \mathrm{GPa}$, then $C 2 c b-24$ at $94 \mathrm{GPa}$, and finally Cmca-24 at $\sim 110 \mathrm{GPa}$. But, the enthalpy differences responsible for the stability of $P b c a-24$ and $C 2 c b-24$ with respect to the high-symmetry Cmca-24 phase are smaller than our calculational accuracy (less than $1 \mathrm{meV} /$ atom).

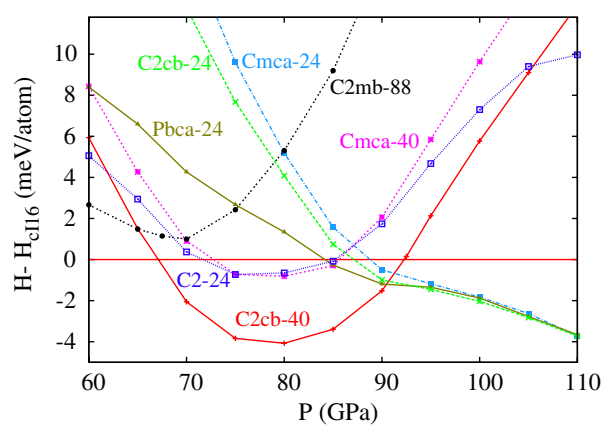

FIG. 2 (color online). Enthalpy differences (relative to the cI16 structure of $\mathrm{Li}-\mathrm{V}$ ) as a function of pressure for the 24atom structures of Li-VIII (Cmca-24, C2cb-24), the 40-atom structures of Li-VII (Cmca-40 and C2cb-40), the calculated $C 2 m b-88$ structure of $\mathrm{Li}-\mathrm{VI}$, and the previously proposed structures $\mathrm{Pbca}-24$ and $\mathrm{C} 2-24$.

The inclusion of the vibrational energies might stabilize the Cmca-24 phase and explain why the other two phases are absent in the experimental sequence, which is obtained at finite temperature.

The calculated diffraction intensities of the $C 2 c b-40$ structure showed good agreement with the experimentally observed intensities estimated on a scale of strong, medium, weak, and unobserved - the data could not be standardly reduced to integrated intensities because of the poorer crystal quality [16]. Nonetheless, these data were sufficient to show that the Cmca-40 structure gave an evidently poorer fit; in particular, two reflections calculated to be clearly visible in $\mathrm{Cmca}$ were unobserved. We thus conclude that the structure of $o C 40$ has space group $C 2 c b$, with atoms located on five $8 b$ sites. Calculations of the full phonon dispersion curves of the $C 2 c b-40$ structure confirm its dynamical stability (see [16]).

The electronic density of states and band structure of the predicted $C 2 c b-40$ structure are shown in Fig. 3 and reveals the material to be a semiconductor with an indirect band gap which increases from $0.82 \rightarrow 1.15 \mathrm{eV}$ between 75 and $95 \mathrm{GPa}$. The gap is much larger than for any other candidate structure. (The hypothetical C2-24 structure [9] had the largest previously predicted gap at $0.276 \mathrm{eV}$.) This supports the general hypothesis that band gap opening helps to stabilize high-pressure structures in simple metals [2].

The semiconducting character of the $C 2 c b-40$ structure can be rationalized from a topological analysis of both the electron localization function (ELF) and electron density. The structure is shown in projection down the $a$ axis in Fig. 4, and can be described in terms of six modulated layers $\left(A B C A^{\prime} B^{\prime} C^{\prime}\right)$ lying perpendicular to the $b$ axis, where the atomic positions in the primed and unprimed layers are related by the $C$-centering translation. $B\left(B^{\prime}\right)$ layers contain the $\mathrm{Li} 2$ atoms, while the $A\left(A^{\prime}\right)$ and $C\left(C^{\prime}\right)$ layers contain a mix of the other Li atoms.

At $85 \mathrm{GPa}$, the shortest in-layer $\mathrm{Li}$-Li distance is $1.604 \AA$ compared with $1.783 \AA$ for the interlayer distance. The ELF topological analysis reveals the existence of three 

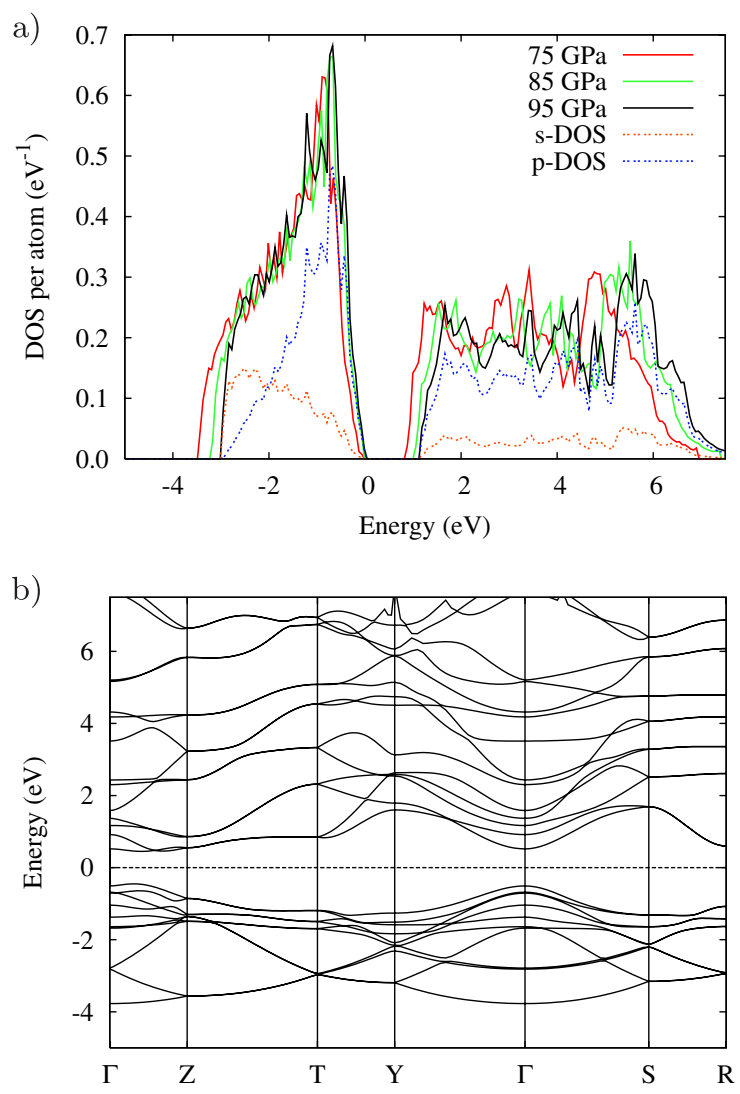

FIG. 3 (color online). (a) Electronic DOS of $C 2 c b-40$ at 75, 85 , and $95 \mathrm{GPa}$. Partial $s$ and $p$ electronic DOS at $95 \mathrm{GPa}$ are also plotted. The zero of energy is placed at $E_{F}$. (b) Electronic band structure of $C 2 c b-40$ at $78 \mathrm{GPa}$, showing an indirect band gap of $0.90 \mathrm{eV}$ along the $\Gamma-Z$ line $\left[\Lambda_{1}(0,0,0.152)-\Lambda_{2}\right.$ $(0,0,0.217)]$, similar to the direct band gap of $1.03 \mathrm{eV}$ at $\Gamma$.

distinct ELF attractors (non-nuclear maxima of the electron density) located on $8 b$ sites at $M 1(0.281,0.040$, $0.813), M 2(0.071,0.757,0.928)$, and $M 3(0.206,0.049$, 0.436) with very high ELF values of 0.961, 0.947, and 0.896 , respectively. As with the atomic arrangement, the ELF attractors [Fig. 4(b)] are located on distorted layers $\left(D E F D^{\prime} E^{\prime} F^{\prime}\right)$ perpendicular to the $b$ axis. The $E\left(E^{\prime}\right)$ layer, which contains the $M 2$ maxima, coincides with the atomic $B$ layer, while the other two layers, $\left(F, D^{\prime}\right)$ or $\left(F^{\prime}, D\right)$ containing both $M 1$ and $M 3$, lie between the $\left(C-A^{\prime}\right)$ or $\left(C^{\prime}-A\right)$ atomic layers.

The ELF analysis suggests that $\mathrm{Li}$ becomes nonmetallic at high pressure by localization of the electrons in interstitial regions. The band structure in Fig. 3(b) suggests a (folded) parabolic free electron band combined with very flat bands below the Fermi energy corresponding to the localized bands in the interstitial regions. This is similar to the electride behavior of the $h P 4$ phase of $\mathrm{Na}$ and $\mathrm{K}$ [3]. Integrating the electron density within the $M 1, M 2$, and $M 3$ ELF attractors gives "pseudoanions" holding $\sim 2,2$, and 1 electrons, respectively. Unlike the almost independent pseudoanions found for the $h P 4$ phases of $\mathrm{Na}$ and $\mathrm{K}$, the ELF attractor basins of Li are connected (Fig. 5), generating
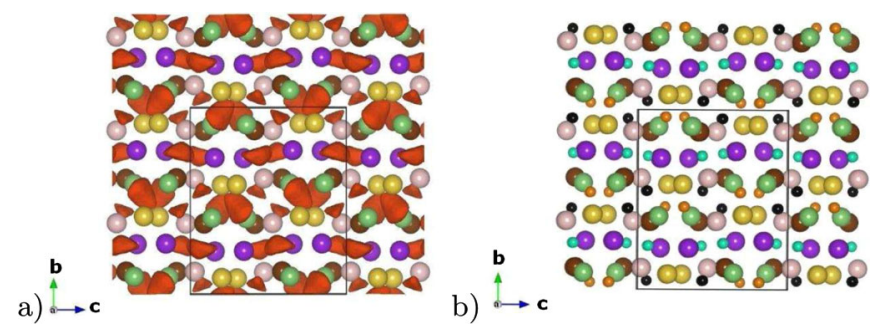

FIG. 4 (color online). (a) ELF $=0.855$ isosurface for $C 2 c b-40$ at $85 \mathrm{GPa}$. Around the Li1-Li5 atoms the ELF isosurface is almost spherical (online: yellow, purple, pink, green, and brown, respectively). The other (nonspherical) surfaces (red online) are associated with interstitial ELF attractors. (b) Crystal structure with atoms colored as above and the ELF attractors represented as small spheres, $\mathrm{M} 1$ and $\mathrm{M} 3$ in layers $D, D^{\prime}, F, F^{\prime}, \mathrm{M} 2$ in layers $E, E^{\prime}$, colored orange, black, and blue, respectively, online.

a complicated framework. In particular, the $M 1$ and $M 3$ ELF attractors merge into a single $M 1-M 3-M 3-M 1$ superbasin containing $\sim 6$ electrons [Fig. $5(\mathrm{~d})]$ within which the ELF never drops below 0.83 .

These ELF basins do not span space; they are separated by regions with ELF values as low as 0.4. Although interstitial electron localization has been observed for other proposed Li phases [9], the fact that all the ELF basins hold electron pairs, in analogy with the electride $h P 4$ phases of $\mathrm{Na}$ and $\mathrm{K}$, is unique to the $o C 40$ structure. This localization of integer numbers of electrons in unconnected pockets provides a powerful real-space explanation for the nonmetallic behavior of $o C 40$. By contrast, the $C 2 c b-24$ structure at $90 \mathrm{GPa}$ has three nonequivalent ELF attractors $\left(M 1^{\prime}, M 2^{\prime}, M 3^{\prime}\right)$ on $8 b, 8 b$, and $4 a$ sites with high ELF values of $0.908,0.888$, and 0.854 containing $1.24,1.07$, and 1.23 electrons, respectively (see [16]).

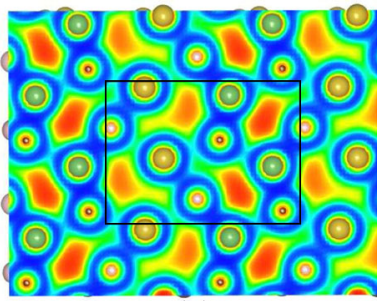

(a)

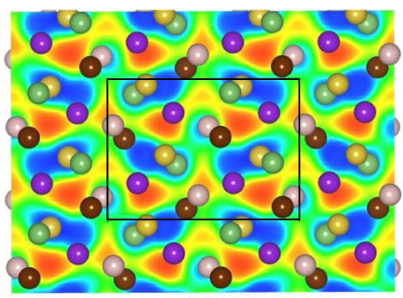

(c)

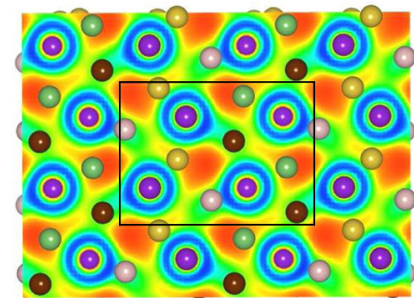

(b)

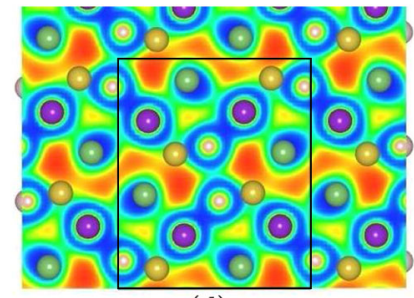

(d)
FIG. 5 (color online). ELF profile of $C 2 c b-40$ on (a) layer $A$, (b) layer $B$, (c) midway between $A$ and $C$ layers, (d) ELF profile on the 400 plane, showing the connection between $M 1$ and $M 3$ ELF attractors. ELF values run from 0 at localized dark rings (blue online) to 1 in gray interstitial patches (red online). Atoms are represented by spheres. 
Although a case can be made for electron pairs lying in $M 2^{\prime}-M 2^{\prime}$ superbasins $(E L F=0.88)$, the metallic character seems to be related to a fractional occupancy of the ELF basins. So, in the metallic Cmca-24 phase at $125 \mathrm{GPa}$ we identify two basins centered on $8 d(\mathrm{ELF}=0.91)$ and $8 f$ $(\mathrm{ELF}=0.89)$ sites containing 1.28 and 1.60 electrons, respectively. Fractional occupancy means that Li-VIII is a metal, but the high ELF value of the basins, and the considerably lower values in between, implies poor conduction and atypical metallic behavior [17].

This real-space analysis complements the $k$-space picture of Fermi-surface-Brillouin-zone interactions, also leading to commensurate charge density modulation. Alternate pictures include projection of the electrons onto atomcentered or two-centered molecular orbitals. The projection onto $s$ and $p$ states in Fig. 3(a) shows that complex behavior occurs at an energy where $2 p$ orbitals can hybridize with $2 s$, and appears to show that both the valence and conduction bands' edges have strong $p$ character. However, given that the electron density lies primarily in the interstitial regions, the value of an interpretation in terms of atom-centered projections seems limited. Previous work has also attributed the interstitial charge density to "pairing" of atoms into "dilithium" molecules, or to $p \pi$ bonding extending into the interstitial regions $[1,6]$. Although the wave functions can be expanded in such basis sets, these pictures contrast strongly with our ELF-based electride picture of welldefined Lewis pairs centered on interstitial sites.

Finally, we address the most complex phase- $o C 88(\mathrm{Li}-$ VI). Systematic absences determined the space group as either $C m m a$ or $C 2 m b$, and the lattice parameters at $65 \mathrm{GPa}$ and $240 \mathrm{~K}$ refined to $a=8.445(5) \AA, b=$ 9.175(11) $\AA$, and $c=8.280(6) \AA$. We have carried out AIRSS, constrained by the experimental information, with 44 atoms per primitive cell. The lowest-enthalpy structure identified by AIRSS had space group $C 2 m b$ (no competing solutions with the Cmma space group were found), and is in good agreement with observed intensities estimated (as for $o C 40$ ) on a scale of strong, medium and weak and unobserved, comparable to the fit obtained for $o C 40$. For details of the calculated atomic coordinates, density of states, and a drawing of the structure, see [16]. The shortest Li-Li distance is $1.77 \AA$. The density of states is low at the Fermi energy but shows no band gap, in agreement with the observed metallic nature of this phase [8]. This structure is the second most stable of the phases calculated at $0 \mathrm{~K}$ and in the Li-VI experimental pressure range, from 60 to $65 \mathrm{GPa}$, Li-V (cI16) has lower enthalpy (Fig. 2). The difference is small, and the inclusion of dynamical effects, both zero-point and finite-temperature, could stabilize this $o C 88$ structure against $c I 16$. Thus AIRSS has revealed the structure, but some additional physics is needed to explain the thermodynamic stability of $o C 88$. A peculiarity of the observed $o C 88$ phase at very low temperature is the reported huge increase of resistivity at $25 \mathrm{~K}$ (4 orders of magnitude) across its narrow range of stability, interpreted as a phase mixture [8]; no such mixture is observed in our experiment, albeit at higher temperatures, and we cannot exclude the possible instability of $o C 88$ to a soft-phonon transition at very low temperatures.

In summary, we have shown that $o C 88$ is metallic with a large, complex structure with probable $C 2 m b$ symmetry; $o C 40$ has a $C 2 c b-40$ structure not observed previously, which is nonmetallic with a larger band gap than any previously found or proposed for $\mathrm{Li}$ at any pressure; and the $o C 24$ phase has the Cmca-24 structure proposed by Rousseau et al. [6]. All of these phases are characterized by the valence electrons occupying interstitial regions, which may become disjoint leading to semiconducting electride phases. This invites further investigation of the role of interstitial electron density in these systems [18].

We thank S. Evans of the ESRF and M. Guthrie, S. V. Sinogeikin, and H.-K. Mao for their help with the experiments, and EPSRC for support. Computer resources were provided by MALTA (CSD2007-00045 and CTQ200914596-C02-02 projects) and the Edinburgh Compute and Data Facility (ECDF).

Note added in proof.-After our Letter was submitted a paper by Lv et al. [19] was submitted to Physical Review Letters describing a similar calculation of the $o C 40$ phase.

[1] J.B. Neaton and N.W. Ashcroft, Nature (London) 400, 141 (1999).

[2] G. J. Ackland and I. R. MacLeod, New J. Phys. 6, 138 (2004).

[3] M. Marqués et al., Phys. Rev. Lett. 103, 115501 (2009).

[4] L. F. Lundegaard et al., Phys. Rev. B 80, 020101(R) (2009).

[5] M. Hanfland, K. Syassen, N.E. Christensen, and D. L. Novikov, Nature (London) 408, 174 (2000).

[6] R. Rousseau, K. Uehara, D. D. Klug, and J. S. Tse, Chem. Phys. Chem. 6, 1703 (2005).

[7] Y. Ma, A. R. Oganov, and Y. Xie, Phys. Rev. B 78, 014102 (2008).

[8] T. Matsuoka and K. Shimuzu, Nature (London) 458, 186 (2009).

[9] Y. Yao, J.S. Tse, and D. D. Klug, Phys. Rev. Lett. 102, 115503 (2009).

[10] C. J. Pickard and R. J. Needs, Phys. Rev. Lett. 102, 146401 (2009).

[11] The authors refer to the conventional setting Aba2 (space group 41). We use the alternative $C 2 c b$ setting where $a b c \rightarrow \bar{c} b a$ to be consistent with the $o C 24$ notation.

[12] C. L. Guillaume et al., Nature Phys. 7, 211 (2011).

[13] C. J. Pickard and R. J. Needs, Phys. Rev. Lett. 97, 045504 (2006).

[14] M. I. McMahon et al., Proc. Natl. Acad. Sci. U.S.A. 104, 17297 (2007).

[15] E. Gregoryanz et al., Science 320, 1054 (2008).

[16] See supplemental material at http://link.aps.org/ supplemental/10.1103/PhysRevLett.106.095502 for further calculation and experimental details.

[17] B. Silvi and C. Gatti, J. Phys. Chem. A 104, 947 (2000).

[18] N. W. Ashcroft, Nature (London) 458, 158 (2009).

[19] J. Lv et al., Phys. Rev. Lett. 106, 015503 (2011). 